\title{
Development of a New Poly Silicate Ferric Coagulant and Its Application to Coagulation-Membrane Filtration Hybrid System in Wastewater Treatment
}

\author{
Firoozeh Nateghi, Ho Kyong Shon, Hadi Khabbaz
}

School of Civil and Environmental Engineering, Faculty of Engineering and Information Technology, University of Technology, Sydney (UTS), Broadway, PO Box 123, Sydney, Australia.

\section{Corresponding author:}

Firoozeh Nateghi: (nateghifir@yahoo.com)

\begin{abstract}
Coagulation is one of the effective pretreatment stages in membrane filtration of wastewaters to produce clean water. Using a suitable coagulant can mitigate membrane fouling. Research in this area is currently being focused on development of improved coagulation reagents such as poly silicate ferric (PSiFe), which has a high molecular weight and large number of positive surface charges with high efficiency at low doses. In this study, PSiFe was prepared using the different approaches. These coagulants were characterised by X-ray diffraction and scanning electron microscopy. According to coagulation jar test results when $\mathrm{Fe} / \mathrm{Si}=1$ the best performance was achieved in terms of the turbidity, the total organic carbon (TOC) and UV254 removals.
\end{abstract}

Another aspect is comparison of the old-PSiFe, FeCl3 and new-PSiFe showed hat in a membrane filtration system using the new-PSiFe not only reduces the required transmembrane pressure (TMP) due to lower fouling, but also improves the TOC removal efficiency.

Keywords: Coagulation, Ploy Silicate Ferric, Membrane Fouling, Membrane Filtration, Transmembrane Pressure 


\section{Introduction}

Water is not an infinite resource in many countries across the world. To have a sustainable and clean water resource, wastewater can be viewed as a resource if it is treated to remove pollutants. Some wastewater professionals are reusing treated wastewater and have found it to be a reliable alternative water source even for drinking purpose. Most of the wastewaters are usually polluted with inorganic and organic particles, as well as contaminants associated with these particles in dissolved forms. The removal of these pollutants is essential as their presence deteriorates water quality. If the treatment complies with applicable and appropriate rules, treated wastwater can be used for many beneficial purposes such as landscape irrigation, agricultural irrigation, aesthetic uses, groundwater recharge, industrial uses, fire protection, household uses and drinking purpose.

Coagulation is one of the most important purification processes in water and wastewater treatment. It is applied to destabilisation and then sedimentation of colloidal particles and dissolved contaminants in water. Traditionally iron and aluminum salts are used as coagulants then an improved coagulant such as poly metal salts (e.g. poly ferric or poly aluminum chloride or sulfates) are being used. As residual aluminium is believed to be harmful to human and other living organisms, iron based coagulants have attracted more interest and attention $[1,2]$.

A new type of coagulation reagent, known as Poly Inorganic Flocculants (PIFs) such as Poly Silicate Ferric (PSiFe), which has a higher charge and molecular weight than the previously used polyvalent metal salts and therefore a higher coagulation ability is currently being investigated by many researchers (References required). Due to the complexity nature of the silica used in the preparation of PSiFe, various preparation methods were proposed. It can be noted that each preparation method may give different performances under the same name of PSiFe. New research requires for quantifying suitable silica characteristics and improving the method of preparation of PSiFe to be employed as an effective coagulant $[3,4]$. Different methods were considered 


\section{The 5th CESE Conference}

9 - 13 September 2012, Melbourne, Australia

to optimise $\mathrm{Si} / \mathrm{Fe}$ ratios, basicity factors, the heating temperature and the aging time.

Most researchers followed one of the currently available procedures using an inorganic or an organic acid or a modifier such as Poly Ferric Sulphate (PFS) or Poly Ferric Chloride (PFC) as polymerisation agent. In many developed methods these additions were made at very low pH (around 2) [3, 5, 6]. There is not any thorough report considering all the above aspects together in the PSiF preparation method. To the best knowledge of the authors, there is no evidence that researchers have precisely investigated the methodology of the polymerisation of silica with alternative acidification using acidic resin to produce improved aggregation characteristics and reach the main goal in particles sedimentation.

In this study, all these factors including initial concentration of silica, acidification method, temperature and suitable time of aggregation of turbidity particles, speed and optimum $\mathrm{pH}$ for $\mathrm{Fe}$ addition have been investigated. Another original aspect of this research is the use of PSiFe coagulants in a hybrid system consisting of coagulation and a submerged membrane to determine the efficiency of this coagulant (PSiFe) on the performance of the membrane, such as membrane fouling. All these factors directly or indirectly would result in having minimum consumption of the materials and high charge neutralization ability, bridging of the particles and all necessary characteristics for optimisation of coagulation. Consequently a steady, reliable and durable membrane treatment system will be developed. The outcome of this research will provide a sustainable coagulation/membrane filtration system with lower chemical consumption, lower waste and minimum operation and maintenance cost.

The main objective of this research is to develop an efficient PSiFe based coagulant with the optimum $\mathrm{Si} / \mathrm{Fe}$ ratio, reducing membrane fouling and improving the functioning of a coagulation/ membrane filtration system in wastewater treatment process.

\section{Experimental Program}




\section{The 5th CESE Conference}

9 - 13 September 2012, Melbourne, Australia

An array of experiments was conducted in this study. In this section preparation details and characterisations of PSiFe are described. In addition, jar test based coagulation experiments as well as the details of a bench scale hybrid system of coagulation-membrane filtration are explained.

\subsection{Preparation of PSiFe}

In the first step, PSiFe was prepared pursuing an available procedure [3]. The procedure involves polymerisation of diluted water glass solution $(6 \% \mathrm{w} / \mathrm{w}$ $\mathrm{SiO}_{2}$ ) with hydrochloric acid followed by metal addition. The PSiFe prepared based on this procedure is called old-PSiFe in this paper. After a detailed literature survey on silica polymerisation methods, the test procedure was improved and a new PSiFe was prepared. This product is called newPSiFe in this paper. The steps used in the preparation of the new-PSiFe are summarised as follows:

1. Dilution of water glass solution to $3 \% \mathrm{SiO}_{2}$

2. Heating the diluted water glass solution to $80-100^{\circ} \mathrm{C}$ for 10 min to improve the aggregation ability

3. Passing the solution through an acidic resin to prepare poly silicate acid (PSiA) without $\mathrm{Na}^{+}$which otherwise causes dispersion instead of aggregation and improves the stability of the solution

4. Heating the PSiA to $80-100^{\circ} \mathrm{C}$ for $10 \mathrm{~min}$ and aging for 24 hours to increase the molecular size of the polymer

5. Adding various amounts of $\mathrm{FeCl}_{3}(1 \mathrm{M})$ to the specific volume of PSiA to prepare four products with different Si/Fe ratios [3, 7, 8, 9,12, 13, 14]

\subsection{Characterisation of PSiFe}

The structure and morphology of PSiFe coagulants were determined using Xray diffraction (XRD), and Scanning Election Microscopy (SEM). The effectiveness of the destabilisation ability of PSiFe was investigated by measuring the zeta $(\zeta)$ potential of the in-situ flocculation product at sedimentation stage [6]. The most important measurement of PSiFe is to determine the proportions of different polymeric species by the ferron-time 
spectroscopy method that has been reported previously by a number of researchers $[4,6,9]$. This analysis has alsobeen conducted in this study.

\subsection{Coagulation experiments (jar test)}

The coagulation experiments were carried out using a jar test apparatus with six paddles. Synthetic wastewater (SW) was prepared with specific contaminants. The jar test consisted of initial 15 minutes rapid mixing (90 rpm) to allow particle destabilization followed by 15 minutes slow mixing for the flocculation period and the final sedimentation period, which lasted 30 minutes. The samples were withdrawn from $20 \mathrm{~mm}$ below the water surface for analysis. The following factors were evaluated in the jar test:

\subsection{Bench scale inline coagulation/membrane hybrid system}

After determining the best $\mathrm{Si} / \mathrm{Fe}$ ratio and the optimum dose of PSiFe by the jar test, a hybrid system, a combination of coagulation and membrane module (hollow fibre), was prepared. This system was used to examine the effect of coagulation with this chemical on the performance of the membrane system for durability and fouling based on a series of standard physical and chemical tests (Fig.1). The analysis involved TOC, turbidity and durability of the critical Transmembrane Pressure (TMP). TMP was measured using a pressure gauge at the outlet of the membrane, TOC was measured after coagulation but before membrane filtration. 


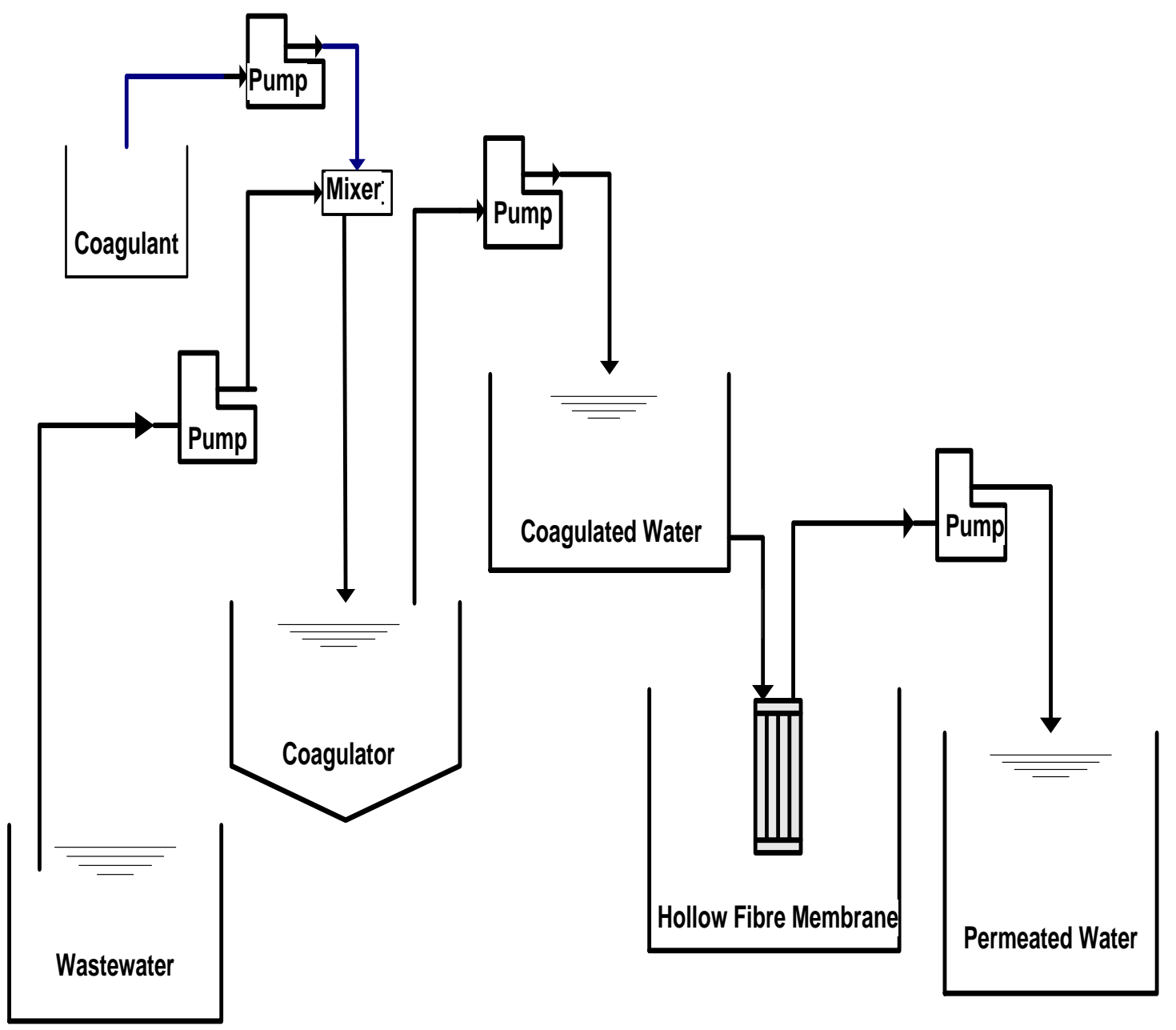

Fig.1. An illustration of the coagulation/ membrane filtration hybrid system

\subsection{Analytic methods}

Turbidity measurement of the suspended solids using a turbidity meter, HACH 2100. Total Organic Carbon (TOC) using MultiN/C 3100 (analytik Jena).

UV254 was used to measure the dissolved organic compounds by UV light absorbance at $254 \mathrm{~nm}$ using Schimadzu UV-1700 Low absorbance corresponds to high removal of dissolved organic matter.

$\zeta$-Potential of flocculated particles at sedimentation stage of jar test to determine the relationshipbetween particle charge characteristics and the tendency for flocculation using a Zeta sizer nanoseries, Malvern Instrument. 
The all measurements on were compared with SW using the following formula:

- \% Removal of TOC,Turbidity,UV $=\frac{\text { Sample Result-SWResult }}{\text { SW Result }} * 100$

\section{Results and Discussion}

\subsection{XRD patterns of PSiFe}

Data acquired from XRD tests showed that the old-PSiFe had large amount of $\mathrm{NaCl}$ impurity whereas the new-PSiFe had no $\mathrm{NaCl}$ crystals. The presence of $\mathrm{Na}^{+}$not only reduced the attachments of the polymer units at aggregation stage but also produced less bridging at final preparation stage. Furthermore, it caused salinity in the treated water. Therefore the new-PSiFe with no salt can be considered a better coagulant.

\subsection{Jar test}

The results of the jar tests for the removal of TOC, turbidity and UV254 at different Si/Fe ratios are summarised in Fig. 2. The results showed that turbidity removal was the highest for $\mathrm{Si} / \mathrm{Fe}=1$ (94\%), TOC removal was the highest for $\mathrm{Si} / \mathrm{Fe}=0.5$ (84\%) and for UV254, Si/Fe $=2$ shows the best performance $(93 \%)$. The main cause of membrane fouling in the coagulation membrane filtration hybrid system is excessive turbidity. Therefore the Si/Fe ratio which produced the greatest turbidity $(\mathrm{Si} / \mathrm{Fe}=1)$ was considered to be the best ratio for the new-PSiFe coagulant. 

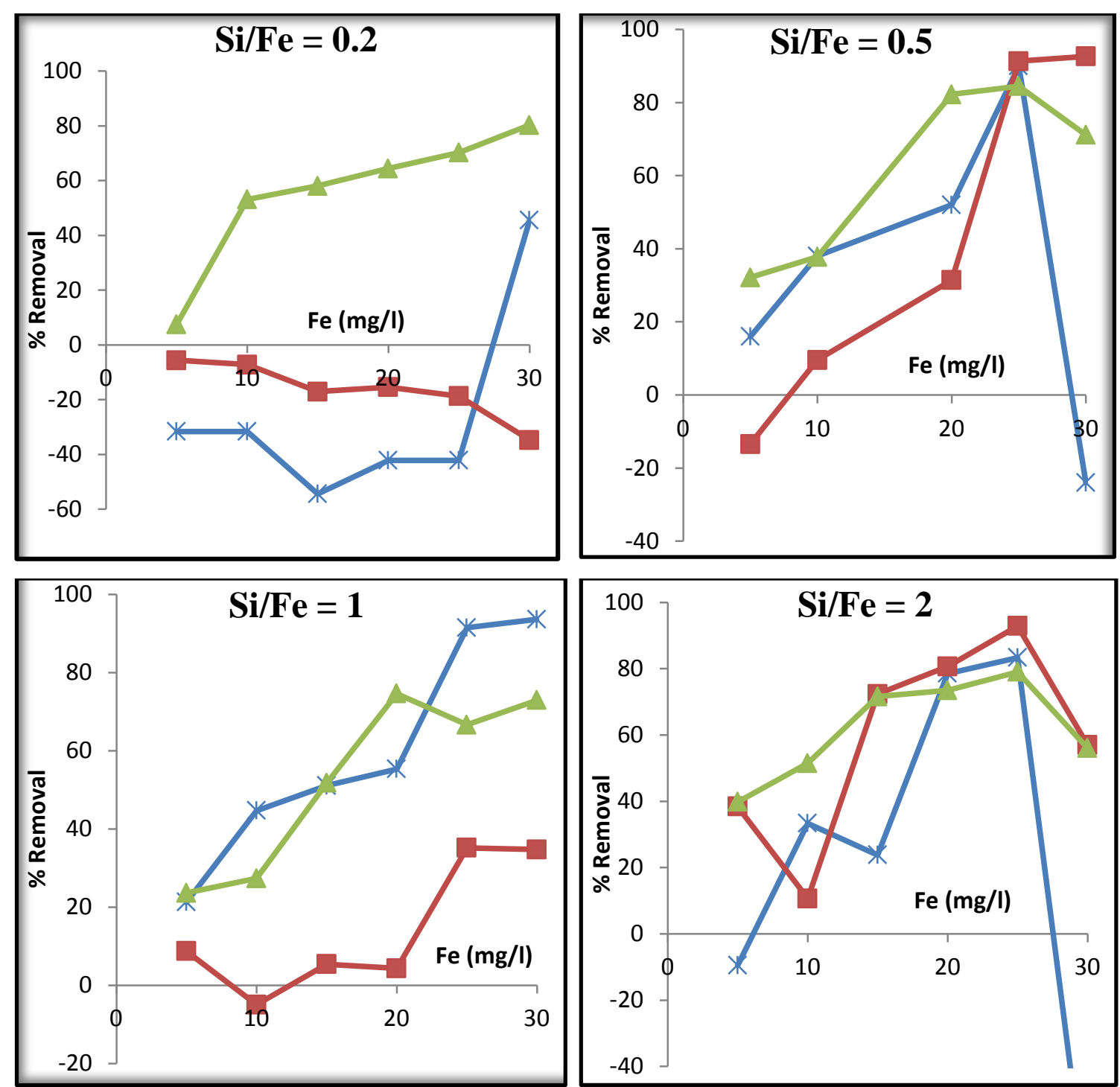

Fig.2.Jar test data for new-PSiFe at different Si/Fe ratios $\quad \boldsymbol{H}$ Turbidity\%, к TOC\%, ${ }_{\mathrm{J}} \mathrm{UV} 254 \%$

\subsection{Bench scale inline coagulation/membrane hybrid system}

Three different coagulants were tested in this experiment. They were $\mathrm{FeCl}_{3}$, old-PSiFe, and new-PSiFe with the ratio of $\mathrm{Si} / \mathrm{Fe}=1$. After the coagulation the treated water was fed to the membrane system. In these experiments cumulative volume of the permeate versus TMP (the difference between inlet and outlet pressure of the membrane) was found for different target coagulants at a dose of $17 \mathrm{Fe}(\mathrm{mg} / \mathrm{l})$ with membrane flux $20 \mathrm{~L} / \mathrm{m}^{2}$.h (Fig.4). 


\section{The 5th CESE Conference}

9 - 13 September 2012, Melbourne, Australia

Furthermore, the TOC removals were compared for the above mentioned coagulants at two sampling points, after coagulation and after membrane filtration (Fig.5).

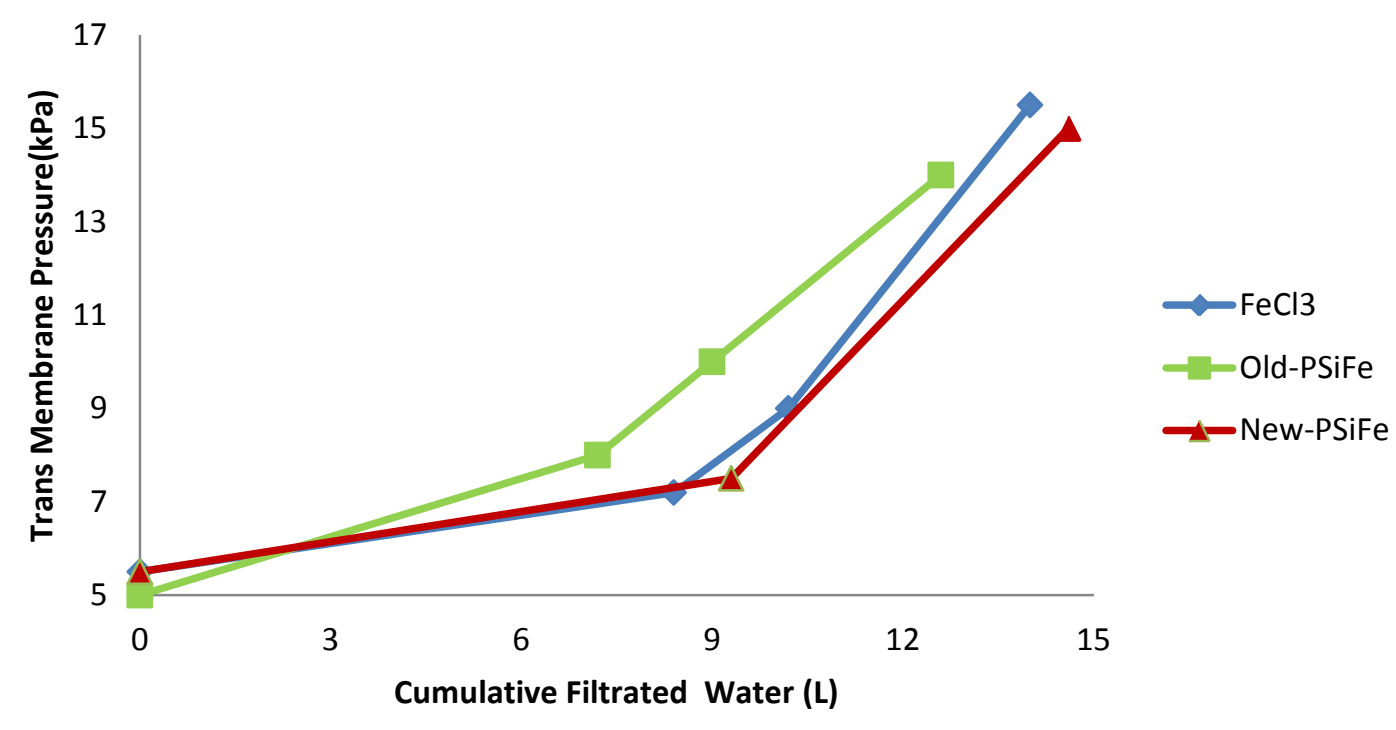

Fig.3. Transmembrane pressure against cumulative membrane outlet volume of treated water for three different coagulants 


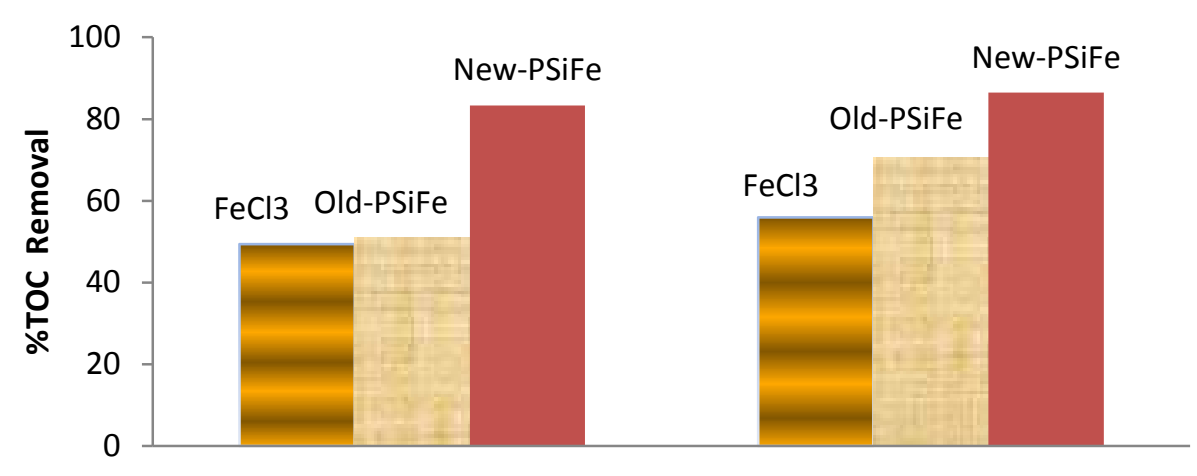

(a) After Coagulation

(b) After Membrane

Fig.4. TOC removal using three different coagulants (a) after coagulation but before passing through the membrane (b) after membrane filtration

Fig.4 shows that new-PSiFe causes lower pressure drop in membrane with high cumulative volume compared to $\mathrm{FeCl}_{3}$ and old-PSiFe. This means that by coagulation with new-PSiFe prior to membrane filtration, we can have more flow through membrane with lower outlet pressure. Fig. 5 shows that the newPSiFe provided the highest TOC removal, three diffenet coagulant are mixed with the specific ratio with wastewater, from two samples are taken before and after the membrane to compare the TOC removal of these coagulants. .

\section{Conclusions}

The main conclusions that can be drawn from this study are:

- The results indicate that the new-PSiFe has superior coagulation efficiency than $\mathrm{FeCl}_{3}$ and a PSiFe prepared based on a common existing method (old-PSiFe).

- Structure and morphology analysis of PSiFe samples carried out using XRD patterns showed that the new-PSiFe had no measurable $\mathrm{NaCl}$ peaks, whereas the old-PSiFe had large amounts of $\mathrm{NaCl}$ crystals. The results confirmed this finding. This point can be explained as $\mathrm{Na}^{+}$can be removed by the acid exchange resin in the preparation of the newPSiFe.

- The new-PSiFe with $\mathrm{Si} / \mathrm{Fe}=1$ is highly efficient in removing the TOC and turbidity and reducing the UV254. Therefore the new-PSiFe 
coagulant provides a lower potential of fouling in downstream membrane filtration treatment.

- The precoagulation with the new-PSiFe has higher effect on the phycochemical factors of membrane filtration such as TMP reduction and TOC removal compared to traditional coagulants ( $\mathrm{FeCl}_{3}$ and oldPsiFe).

\section{Acknowledgements}

This project has been conducted under the auspices of the Australian Research Council (ARC) with funding provided by ARC Discovery Project DIP 0986027. Prof S. Vigneswaran's academic and technical advice is also gratefully acknowledged.

[1] J.Orme, E.V.Ohanian, 1993, US Environmental Protection Agency, Washington, D. C.

[2] T.P.Falten, 2001, Aluminum as arisk factor in Azheimer's disease, with emphasis on driniking water, Brain Research Bulletin. 55,187-196.

[3] D.Wang, H.Tang, 2001, Modified inorganic polymer flocculant PFSi: its preparation, characterisation and coagulation behavior. Water. Res. 35,34183428.

[4] A.I.Zouboulis, P.A.Moussas, 2008, Polyferric silicate sulphate (PFSiS): Preparation, characterisation and coagulation behaviour. Desalination 224,307-316

[5] X.Xu, S.Yu, W.Shi, Z.Jiang, C.Wu, 2009, Effect of acid medium on the coagulation efficiency of polysilicate-ferric (PSF)- A new kind of inorganic polymercoagulant. Separation and Purification Technology 66, 486-491.

[6] ] A.I.Zouboulis, P.A.Moussas, 2008, A study on the properties and coagulation behaviour of modified inorganic polymeric coagulant- Polyferric silicate sulphate(PFSiS), Separation and Purification Technology 63, 475-483

[7] R.K.Iler, R.Dalton, 1956, Degree of hydration of particles of colloidal silica in aqueous solutionGerasselli Chemical Department

[8] R.K.Iler, 1979, The Chemistry of Silica: Solubility, Polymerization, Colloid and Surface Properties and Biochemistry of Silica. 
[9] J.D. Mahulkar, Y.Wang, 2006, Fumed silica to colloidal silica conversion process .U.S patent No. 2006/0283095.

[10] T.Hasegawa, T.Onitsuka, Y.Ehara, K.Hashimoto, H.Akazawa, 1993, Method for prodution locculant of water treatment. (1993) U. S. Patent No.5,227,089

[11] W.O.Roberts, 2005, Manufacturing and application of water -brone colloidal silica,CRC Press, 131-175.

[12] B.Gao, B.Lio, T.Chen, Q.Yue, 2011, Effect of aging period on the characteristics and coagulation behaviour of poly ferric Chloride- Poly amid composit coagulant for synthetic waste water treatment, J. Hazardeous Material 187,413-420.

[13] T.Yokoyama, T.Nakazato, T.Tarutani, 1980, Bull, Chem. Society 53,850853.

[14] M.J.Schick, 1998, Colloidal Silica: Fundamentals and Applications, Plenum Press, New York, pp. 131-147. 